\title{
Pathogenesis of alpha-I antitrypsin deficiency in the liver: new approaches to old questions
}

\begin{abstract}
Alpha-1 Antitrypsin deficiency (A1ATD) can progress to cirrhosis and hepatocellular carcinoma; however, not all patients are susceptible to severe liver disease. Liver transplantation is the only cure for A1ATD-related liver disease. A1ATD is caused by a toxic gain-of-function mutation in the human SERPINA1 gene, generating mis folded ATZ protein "globules" in hepatocytes. These insoluble aggregates overwhelm protein clearance pathways and lead to chronic intracellular stress. This review serves to summarize the basic hepatic mechanisms involved in A1ATD, as described in relevant in vitro and animal models. Potential treatments such as autophagy-enhancing agents and molecular therapies are also discussed. Clinical trials are underway to further assess some of these novel approaches in patients, but more safety and efficacy data is needed to successfully translate these interventions from the laboratory to the clinic.
\end{abstract}

Volume 2 Issue 2 - 2016

\author{
Zahida Khan \\ Division of Pediatric Gastroenterology, Hepatology and \\ Nutrition, Children's Hospital of Pittsburgh, USA
}

\begin{abstract}
Correspondence: Zahida Khan, Children's Hospital of Pittsburgh of UPMC, Division of Pediatric Gastroenterology, Hepatology and Nutrition 440I Penn Avenue, Faculty Pavilion 6th Floor, Pittsburgh, Pennsylvania, USA, I5224-1334, Tel (4I2) 6925180, Fax (412) 6927355, Email zahida.khan@chp.edu
\end{abstract}

Received: March II, 2016 | Published: May 17, 2016

Keywords: PIZ mouse, autophagy, ATZ globule, carbamazepine, C. Elegans, liver transplantation, PASD, SERPINA1, TFEB

Abbreviations: A1ATD, alpha-1 antitrypsin deficiency; A1AT, alpha-1 antitrypsin; ER, endoplasmic reticulum; PAS, periodic acid schiff; SNPs, single nucleotide polymorphisms; ERManI, ER mannosidase I; IPSC, induced pluripotent stem cell; GC, globulecontaining; GD, globule devoid; 4-PBA, 4-phenylbutyric acid; GFP, green fluorescent protein; LOPAC, library of pharmacologically active compounds

\section{Introduction}

At the time of this writing, there are over 15,000 patients awaiting liver transplantation in the USA, but only 6729 liver transplants were performed last year. ${ }^{1}$ Alpha-1 Antitrypsin Deficiency (A1ATD) is the most common inherited cause of pediatric liver disease and transplantation. Liver transplantation remains the only treatment for patients with severe liver disease; however, it is difficult to predict which factors predispose some patients to develop liver disease while sparing others. For example, prospective studies of a Swedish cohort of 127 A1ATD patients identified by mass neonatal screening reported that $\sim 8 \%$ of homozygotes develop clinically significant liver disease over their lifetime..$^{2-4}$ This suggests the role of other genetic and/or environmental modifiers of disease susceptibility.

The classical form of A1ATD is an autosomal co-dominant disorder that affects as many as 1 in 3000 live births in the United States and Europe. ${ }^{5}$ Normal human Alpha-1 Antitrypsin (A1AT) is a $52-\mathrm{kDa}$ glycoprotein of the serpin family, predominantly produced in the liver and released into the blood. In affected patients, circulating levels of mutant $\leq 15 \%$ A1AT of normal are protein levels. Serum A1AT acts as an acute phase reactant and it functions to inhibit destructive neutrophil elastase in the lung; therefore, deficiency of the serum protein leaves the lungs exposed to damage. Mutant ATZ leads to respiratory cell proteinopathy and fibrosis later in life. ${ }^{6}$

The most common genetic defect in A1ATD involves homo zygosity for the $\mathrm{Z}$ allele (ZZ, causing Pi*ZZ phenotype) in the SERPINA1 gene on human chromosome. This single base pair substitution encodes a glutamate lysine mutation at position $342 .{ }^{7}$ This change in negative- to-positive charge results in a toxic gain-of-function mutation (ATZ), leading to conformational changes in the secondary and tertiary structure of the protein. Mis folded ATZ monomers accumulate in the Endoplasmic Reticulum (ER) of hepatocytes, forming insoluble globules that are Periodic Acid-Schiff (PAS)+/diastase-resistant. ER protein degradation pathways, involving either autophagy or proteasomes, become overwhelmed. As the ATZ globules accumulate in the liver, chronic hepatocyte injury from ER stress, mitochondrial damage, and impaired protein clearance can progress to cirrhosis and hepatocellular carcinoma in some individuals. ${ }^{8}$ Modifiers of disease susceptibility are therefore a focus of active investigation, especially in the age of "big data". Single nucleotide polymorphisms (SNPs) in the SERPINA1 gene have been identified in children with PiZZ phenotype and moderate-to-severe liver disease. ${ }^{9}$ Critical ER proteins that control ATZ degradation include the transmembrane chaperone calnexin and the enzyme ER Mannosidase I (ERManI), both of which have been implicated in reduced efficiency of degradation using cell lines derived from PiZ patients susceptible to liver disease. ${ }^{10-13}$ These molecular findings are promising, but also highlight the question of how these biological processes can be harnessed to aid in the treatment and prognosis of A1ATD liver disease. Several laboratory models have been developed to further explore these pathways.

\section{Disease models of AIATD liver disease}

Traditional models of A1ATD range from stably transfection cell lines to transgenic mice. Although these models and well-characterized, they may not efficiently fulfil the growing need for multiplex drug screening and personalized pharmacogenetics. To expedite translation of new potential therapies to the clinic, recent generation of effective rapid screening models have now been established. As personalized medicine evolves, complementary models of metabolic liver disease, such as patient-derived induced pluripotent stem (IPS) cells and mice with "humanized" livers, are in the pipeline, and will be innovative for the study of patient-specific variations and disease phenotypes in A1ATD. The primary models of A1ATD liver disease in the literature are reviewed below. 


\section{In vitro models of AIATD}

Mammalian cell lines have been used extensively to study the complex intracellular processing of mis folded ATZ proteins. Detailed sub cellular analysis in mouse hepatoma cell lines transfection with human ATZ expression vectors revealed that degradation of mutant ATZ proteins localizes within ER-to-Golgi vesicle trafficking in the early secretory pathway. ${ }^{14}$ Proteostasis is a dynamic process. Although insoluble macromolecular complexes form ATZ aggregates, smaller soluble forms do exist within the ER where they are subject to degradation pathways, possibly playing a role in maintaining protein quality control. ${ }^{15} \mathrm{As}$ in PiZ mice, treatment of ATZ-expressing human cell lines with autophagy-enhancing agents reduced ATZ aggregates. ${ }^{16}$ The opposite effect is observed when treating the same cells with inhibitors of autophagy, or when studying autophagy-deficient cell lines and yeast strains..$^{17,18}$

More recent work has focused on induced pluripotent stem cell (IPSC) lines as a method of modelling a wide range of monogenic metabolic liver diseases, including A1ATD. Direct reprogramming of somatic cells from PiZ mice was the first "proof-of-principle" step in developing an IPSC model. ${ }^{19}$ The technique was soon adapted to patient-derived iPSCs that could be differentiated into hepatocytes, allowing a novel platform to study variations in A1ATD disease modifiers and phenotype..$^{20}$ Tafaleng et al. ${ }^{20}$ demonstrated that IPSC hepatocytes recapitulate the accumulation and processing of ATZ globules found in patients with severe liver disease. ${ }^{20}$ With scaled up differentiation protocols, patient-derived IPSC hepatocytes could also provide an unlimited source for drug discovery and screening, paving the way for personalized medicine. Wilson et al. ${ }^{21}$ recently identified 135 genetic modifiers of severe liver disease via global transcriptome analysis of IPSC hepatocytes. ${ }^{21}$ These cells also showed a favorable autophagy response to autophagy-enhancing agents in culture. Another potential application for IPSC hepatocytes is cellular and gene therapy. ${ }^{22}$

\section{PiZ mouse model of AIATD}

Despite knowing the molecular defect in A1ATD, there are still many unanswered questions about specific modifiers of disease severity and susceptibility. The clinical variability of liver disease in patients with A1ATD may be related to phenotypic differences at the cellular level. In PiZ hepatocytes, the balance of proteostasis, including autophagy and other protein degradation pathways, influences susceptibility to or protection from toxic ER stress. ${ }^{4,23}$ Histologically, there are two main hepatocyte subpopulations in A1ATD, described as "globule-containing" (GC) or "globule-devoid" (GD) cells (Figure 1), with distinct characteristics.

From a cell biology perspective, the differential capabilities of GC and GD hepatocytes have been described in the well-established PiZ transgenic mouse strain, which over-expresses human ATZ (note that "PiZ" refers to having 2 copies of the transgene). The transgene consists of multiple genomic fragments of DNA that contain the coding regions of the human ATZ gene together with introns and $\sim 2$ kilo bases of upstream and downstream flanking regions. ${ }^{24}$ The transgene is expressed primarily in liver, making this a pure toxic gain-of-function model of hepatocyte ER stress. ${ }^{24,25}$ Minor levels are also found in other tissues known to express A1AT. Of note, the wildtype murine A1AT is still expressed in this model; therefore, serum levels of endogenous A1AT are preserved and the lungs are relatively protected.

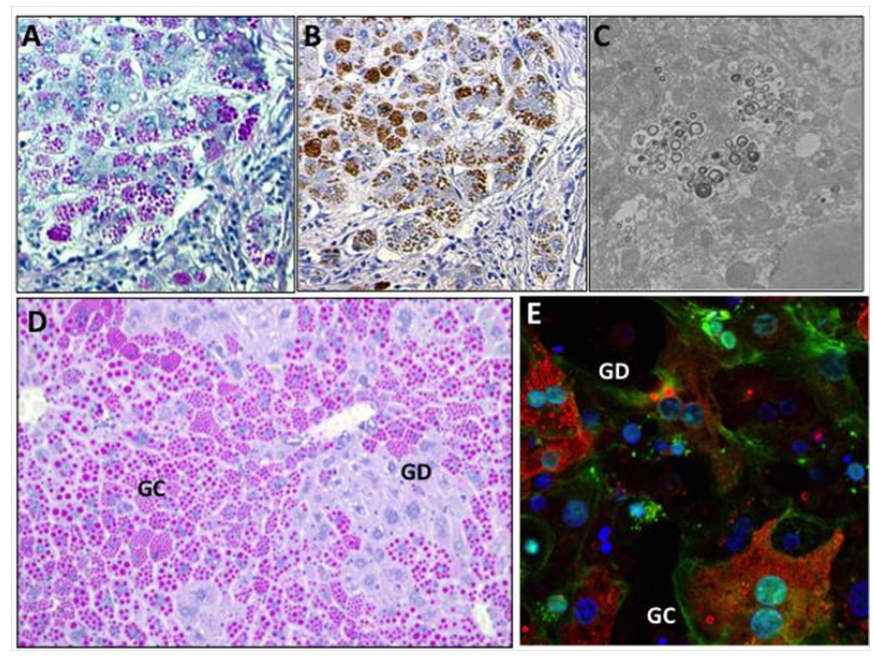

Figure I Liver specimen from AIAT deficiency.

A) PASD stain highlights ATZ globules (pink) in a human explanted liver from a pediatric patient with severe liver disease(100x).

B) AIAT immunohistochemistry of ATZ globules in serial section of $A(100 x)$.

C) EM of pediatric liver explant showing accumulation of autophagolysosomes(15000x).

D) PASD stain of PiZ transgenic mouse liver highlights globule-containing (GC) and globule-devoid (GD) cells(I00x).

E) Con focal immunofluorescence staining of isolated primary $\mathrm{PiZ}$ mouse hepatocytes in culture, showing AIAT (red) globules, DAPI (blue) nuclear stain, and phalloidin (green) actin cytoskeletal stain (200x). In general, hepatocytes contain a range of small, large, intermediate, or no ATZ globules.

In the PiZ transgenic mouse, GC hepatocytes are replaced over time with less stressed GD hepatocytes, making this an ideal model to investigate the differential capabilities of these two cell types (Figure 1D) (Figure 1E). Functionally, PiZZ mice have reduced hepatic glycogen stores and impaired fasting tolerance. ${ }^{26,27}$ At the tissue level, they develop low-grade hepatic inflammation, regeneration, steatosis, and even malignancies as they age, recapitulating the classical liver disease found in A1ATD patients. ${ }^{16,28}$ Mela et al. ${ }^{29}$ analyzed liver biopsies from 60 A1ATD patients with varying degrees of fibrosis, and found significantly larger hepatocyte nuclei and shorter telomere length, both markers of senescence, in A1ATD human hepatocytes compared to age- and sex-matched control liver tissues. ${ }^{29}$ PiZZ patients had significantly more changes than PiZ heterozygotes, suggesting a proteotoxic effect. Similar changes were noted in GC cells compared to adjacent GD cells within the same human tissue sections. These findings were associated with increased patient age, ATZ globule content, and fibrosis.

Several studies have investigated the pathogenesis of A1ATD in detail using the PiZ mouse. Comparable to humans, aging liver tissue from older PiZ mice contains increased reactive oxygen species, lower levels of protective antioxidant enzymes, and evidence of cellular damage when compared to younger mice. ${ }^{30}$ Interestingly, hepatotoxicity from ATZ globule accumulation is more severe in male PiZ mice compared to females, which parallels the disease spectrum found in humans. ${ }^{31,32}$ In fact, testosterone treatment of female PiZ mice increased ATZ expression and hepatocellular proliferation to 
a level comparable with that observed in males. ${ }^{31}$ Rudnick et al. ${ }^{31}$ found that although intracellular accumulation of ATZ globules is associated with a regenerative stimulus, GD cells still demonstrated a proliferative advantage over GC cells. ${ }^{31}$ Similarly, Ding et al.${ }^{33}$ used cell transplantation to demonstrate that wild-type and to some extent GD hepatocytes have a proliferative advantage over GC hepatocytes. ${ }^{33}$ Isolated PiZ mouse hepatocytes are susceptible to decreased proliferation and increased apoptosis with increasing ATZ globule load. ${ }^{34,35}$ Taken together, these studies confirm that GD hepatocytes exhibit increased survival and regenerative capacity in times of stress.

As a preclinical model, the PiZ mouse offers a unique opportunity to study mechanisms of protein clearance of ATZ globules. Impaired autophagy is the major intracellular pathway implicated in A1ATD. During autophagy, autophagosomes engulf cytoplasmic components, such as cytosolic proteins and organelles, marked for degradation. ${ }^{36}$ Although starvation-induced autophagy is an evolutionarily conserved survival mechanism, fasting in PiZ mice leads to poor outcomes and no increase in hepatic autophagy. ${ }^{27}$ Baseline autophagosomes activity is observed in the liver of PiZ transgenic mice and in liver biopsy specimens from patients with A1ATD. ${ }^{37}$ Interestingly, it has been shown that the transcription factor TFEB appears to be a master regulator of autophagy and lysosomal biogenesis. The nutrient-sensing kinase enzyme mTORC1, a regulator of starvation-induced autophagy, phosphorylates cytoplasmic TFEB in fed conditions. ${ }^{38}$ Cytoplasmic TFEB is de phosphorylated in the fasted state, and translocates to the nucleus to induce autophagy-related gene expression. TFEB gene transfer in PiZ mice induced macro autophagy and corrected the liver disease, leading to increased degradation of polymerized ATZ in auto lysosomes and decreased expression of ATZ monomers. ${ }^{39}$

From a clinical perspective, recent studies have focused more on autophagy-enhancing agents to induce ATZ disposal. Rapamycin given in weekly pulses was the first drug reported to increase autophagic activity and reduce intrahepatic accumulation of polymerized ATZ proteins. ${ }^{40}$ Rapamycin is an mTOR inhibitor clinically in use as a potent immuno suppressant, but can have serious complications including lung toxicity. Hidvegi et al. ${ }^{16}$ examined the effects of carbamazepine (CBZ), a well-known FDA-approved anticonvulsant and mood stabilizer with an extensive safety profile, in PiZ mice. ${ }^{16} \mathrm{CBZ}$ mediated a reduction on hepatic ATZ globule load and ameliorated hepatic fibrosis in PiZ mouse liver, making it an ideal therapeutic strategy. Currently, a Phase II/III clinical trial for the use of CBZ in severe A1ATD liver disease is ongoing, with dosing of $1200 \mathrm{mg}$ /day for subjects over 15years of age (ClinicalTrials.gov NCT01379469). Members of the phenothiazine class of drugs, such as fluphenazine, also have combined autophagy-enhancing and mood stabilizing effects. ${ }^{41}$ As with CBZ, fluphenazine treatment in PiZ mice reduced the accumulation of ATZ in the liver and mediated a decrease in hepatic fibrosis, making it another promising agent. ${ }^{41}$ Synthetic bile acids, many of which are in clinical trials for treating other chronic liver diseases, have recently demonstrated potential benefits for inducing autophagic clearance of ATZ globules in PiZ mice. ${ }^{42}$

\section{C. elegans model of AIATD}

It should be noted that identification of many of the above autophagy-enhancing agents would not have been possible without initial automated high-throughput live animal drug screening in roundworms. ${ }^{43} \mathrm{C}$. elegans is a transparent nematode that is $\sim 1-\mathrm{mm}$ in length. Although C. elegans is a simple un segmented pseudocoelomate that lacks respiratory, circulatory, and hepatobiliary systems, it can be genetically engineered to express fluorescently-tagged wild-type (ATM) or Z mutant forms (ATZ) of the human SERPINA1 gene fused to green fluorescent protein (GFP) ${ }^{44}$ These animals secrete soluble ATM, but retain polymerized ATZ protein within dilated ER cisternae. ${ }^{44}$ Transgenic ATZ-expressing worms exhibit slow growth, smaller brood size, and decreased longevity, similar to PiZ mice. This novel C. elegans model has been adapted for screening a library of 1280 compounds in the Library of Pharmacologically Active Compounds (LOPAC), to identify drugs in active clinical use that could be immediately tested in clinical trials and "repurposed" for treating A1ATD-associated liver disease. ${ }^{43}$

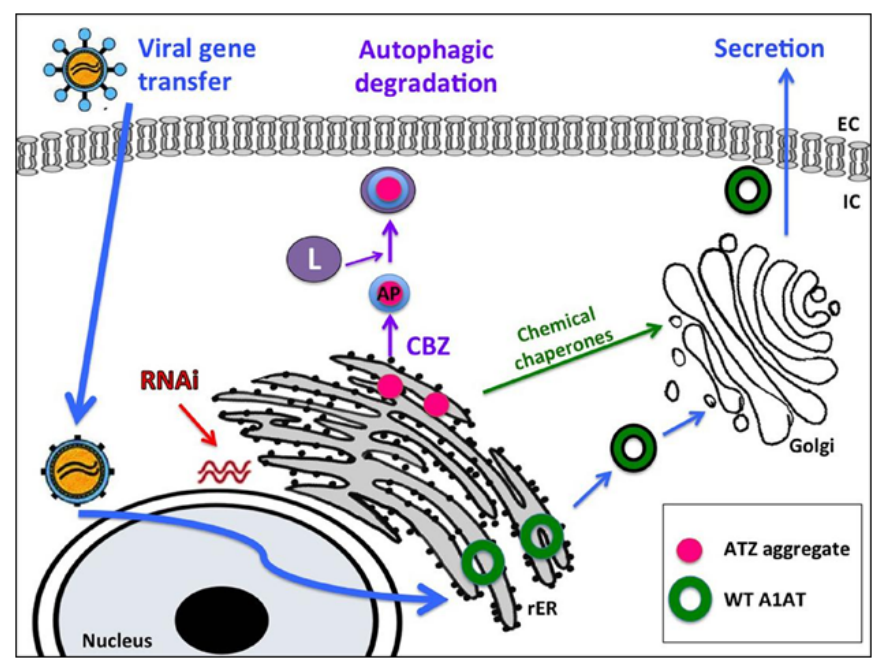

Figure 2 Pathogenesis of liver disease in AIAT deficiency and potential pharmacologic interventions.

Insoluble mutant ATZ protein aggregates accumulate in the hepatocyte endoplasmic reticulum (rER), while wild-type (WT) AIAT proteins are folded and secreted normally from the hepatocyte into the extracellular (EC) space. Viral gene transfer using AAV can introduce the WT AIAT gene into any cell type for expression and secretion of the normal AIAT protein. Autophagyenhancing agents, such as carbamazepine (CBZ), promote intracellular (IC) protein clearance mechanisms such as autophagy, and also to a lesser extent proteosomal degradation, to reduce ATZ aggregates in the liver ( $\mathrm{AP}=$ =autophagosome, $\mathrm{L}=$ lysosome). RNA interference (RNAi) technology knocks down mutant ATZ gene expression at the mRNA level, preventing toxic accumulation of ATZ aggregates. Chemical chaperones are thought to facilitate proper folding of mutant ATZ proteins, so that they can be exported out of the hepatocyte via the secretory pathway.

\section{Molecular therapies for liver disease in A I ATD ${ }^{45-51}$}

Gene therapy for A1ATD liver disease: Several approaches to gene therapy for A1ATD are under investigation (Table 1). One strategy involves targeted "knock-down" of the mutant ATZ gene, which has been studied both in vitro and in PiZ mice. In IPSC hepatocytes derived from patients with severe liver disease, Lentiviral vectormediated expression of short hairpin RNAs (shRNAs) directed against the ATZ led to a $66 \%$ reduction in intracellular ATZ protein, which was functionally relevant and efficiently maintained with hepatocyte differentiation. ${ }^{22}$ This opens up future possibilities for ex vivo targeted gene correction and autologous cell therapy. Recent studies have also focused on anti-sense oligonucleotides to effectively target and reduce human ATZ expression in PiZ mice. ${ }^{52}$ Short-term administration in these animals stopped liver disease progression, while long-term treatment reversed liver disease and decreased fibrosis. Administration in non-human primates led to a 
$\sim 80 \%$ reduction in levels of circulating normal AAT, demonstrating potential for this approach in higher species. ${ }^{53}$ Repeat dosing had long-lasting effects, preventing and even reversing accumulation of ATZ aggregates in PiZ mice and cynomolgus monkeys. ${ }^{54}$ Finally, Micro RNA (MiRNA) "dual therapy" has also been studied in PiZ mice, where effective knock-down of mutant ATZ is accompanied by expression of wild-type A1AT (ATM). ${ }^{55}$ miRNA dual therapy led to decreased hepatic inflammation and ATZ globule accumulation, with concomitant increase in normal serum A1AT. A clinical trial is now under way to further investigate antisense oligonucleotides as a potential therapy for A1ATD liver disease in adult patients (ClinicalTrails.gov, NCT02363946).

Table I Clinical studies listed in ClinicalTrials.gov for therapy of liver disease in AIATD.45

\begin{tabular}{|c|c|c|c|c|c|}
\hline Therapeutic Agent & Therapeutic Mechanism & Methodology & Clinical Trial ID & $\begin{array}{l}\text { Current } \\
\text { Status }\end{array}$ & References \\
\hline Carbamazepine (CBZ) & $\begin{array}{l}\text { Mood stabilizer and anti-epileptic } \\
\text { drug that enhances autophagy to } \\
\text { increase ATZ protein clearance }\end{array}$ & Phase 2, interventional & NCTOI 379469 & Recruiting & 16 \\
\hline ALN-AAT & $\begin{array}{l}\text { RNAi-based knockdown of ATZ } \\
\text { protein expression }\end{array}$ & Phase $\mathrm{I} / 2$, interventional & NCT02503683 & Recruiting & \\
\hline ARC-AAT & $\begin{array}{l}\text { RNAi-based knockdown of ATZ } \\
\text { protein expression }\end{array}$ & Phase I, interventional & NCT02363946 & Recruiting & \\
\hline $\begin{array}{l}\text { rAAVI-CB-hAAT gene } \\
\text { vector }\end{array}$ & & Phase I, interventional & NCT00430768 & Completed & \\
\hline $\begin{array}{l}\text { rAAVI-CB-hAAT gene } \\
\text { vector }\end{array}$ & $\begin{array}{l}\text { AAV gene transfer of normal } \\
\text { AIAT into muscle cells }\end{array}$ & Phase 2, interventional & NCT0I054339 & $\begin{array}{l}\text { Active, not } \\
\text { recruiting }\end{array}$ & $46-49$ \\
\hline $\begin{array}{l}\text { rAAV2-CB-hAAT gene } \\
\text { vector }\end{array}$ & & Phase I, interventional & NCT003774I 6 & $\begin{array}{l}\text { Active, not } \\
\text { recruiting }\end{array}$ & \\
\hline $\begin{array}{l}\text { 4-phenyl butyrate (4- } \\
\text { PBA) }\end{array}$ & $\begin{array}{l}\text { Molecular chaperone to increase } \\
\text { ATZ protein secretion from liver }\end{array}$ & Phase 2, interventional & NCT00067756 & Completed & 50,51 \\
\hline
\end{tabular}

Protein-directed therapy for A1ATD: In addition to enhancing autophagic degradation, proteinopathies can be managed by increasing the endogenous protein (i.e., increasing production, correcting mis folding and increasing secretion) or by replacing with functional exogenous protein. Although A1AT is an acute phase reactant that increases with fever, shock, trauma, or pregnancy, pharmacologic strategies to enhance endogenous production in deficient patients have not proven to be effective. ${ }^{56}$ Though not discussed here, intravenous augmentation using pooled human A1AT is currently the most direct and efficient means of elevating A1AT levels in the plasma and in the lung interstitium in adults. ${ }^{5}$ Another attractive liver-based strategy is the use of chemical chaperones to correct protein mis folding. 4-phenylbutyric acid (4-PBA), used safely as an ammonia scavenger in patients with urea cycle disorders, partially corrected deficient circulating levels of ATZ in PiZ mice. ${ }^{50}$ Increased secretion of ATZ protein was also observed in cultured cells treated with 4-PBA. Despite this reversal of protein mis folding, a randomized controlled trial of oral 4-PBA did not increase serum protein levels in patients, and symptomatic and metabolic side effects were significant. ${ }^{51}$

\section{Summary and future directions}

Since the first description of A1ATD over 50years ago, significant progress has been made in understanding the basic pathobiology of both the liver and lung disease. Despite these achievements, current management primarily involves supportive measures, such as augmentation therapy for lung disease. Transplantation is still the only cure for severe chronic liver and/or lung disease. Recent findings on intracellular protein processing and autophagy have identified novel therapies to prevent liver damage from ATZ accumulation; however, strategies to alter gene expression and protein secretion are still under investigation. Further work is needed comparing both genomewide associations as well as personalized patient-specific variations.
Understanding these mechanisms at the molecular level will help shed light on modifiers of disease susceptibility in patients with A1ATD.

\section{Acknowledgements}

Dr. Khan acknowledges grant support from NIH/NICHD PHS K12HD052892, the Alpha-1 Foundation, and the Hillman Foundation. Dr. Khan also acknowledges assistance with Figure 1 from Dr. Donna B. Stolz (University of Pittsburgh's Center for Biologic Imaging, Core A), as well as from Dr. Sarangarajan Ranganathan (Pathology Department, Children's Hospital of Pittsburgh).

\section{Conflict of interest}

Author declares that there is no conflict of interest.

\section{References}

1. United Network for Organ Sharing (UNOS). United Network for Organ Sharing Transplant Trends, USA. 2015.

2. Piitulainen E, Carlson J, Ohlsson K, et al. Alpha1-antitrypsin deficiency in 26-year-old subjects: lung, liver, and protease/protease inhibitor studies. Chest. 2005;128(4):2076-2081.

1. Sveger T. Liver disease in alpha1-antitrypsin deficiency detected by screening of 200,000 infants. N Engl J Med. 1976;294(24):1316-1321.

2. Ghouse R, Chu A, Wang Y, et al. Mysteries of alpha1-antitrypsin deficiency: emerging therapeutic strategies for a challenging disease. Dis Model Mech. 2014;7(4):411-419.

3. Craig Miller. Liver disease in alpha-1 antitrypsin deficiency. J Liver Res Disord Ther. 2015;1(3):00011.

4. Hidvegi T, Stolz DB, Alcorn JF, et al. Enhancing Autophagy with Drugs or Lung-directed Gene Therapy Reverses the Pathological Effects of Respiratory Epithelial Cell Proteinopathy. J Biol Chem. 2015;290(50):29742-29757. 
5. Ledley FD, Woo SL. Molecular basis of alpha 1-antitrypsin deficiency and its potential therapy by gene transfer. $J$ Inherit Metab Dis. 1986;9(Suppl 1):85-91. s

6. Teckman JH, An JK, Blomenkamp K, et al. Mitochondrial autophagy and injury in the liver in alpha 1-antitrypsin deficiency. Am J Physiol Gastrointest Liver Physiol. 2004;286(5):851-862.

7. Chappell S, Hadzic N, Stockley R, et al. A polymorphism of the alpha1antitrypsin gene represents a risk factor for liver disease. Hepatology. 2008;s47(1):127-132.

8. Pan S, Huang L, McPherson J, et al. Single nucleotide polymorphismmediated translational suppression of endoplasmic reticulum mannosidase I modifies the onset of end-stage liver disease in alpha1antitrypsin deficiency. Hepatology. 2009;50(1):275-281.

9. Qu D, Teckman JH, Omura S, et al. Degradation of a mutant secretory protein, alpha1-antitrypsin $\mathrm{Z}$, in the endoplasmic reticulum requires proteasome activity. J Biol Chem. 1996;271(37):22791-22795.

10. Teckman JH, Perlmutter DH. The endoplasmic reticulum degradation pathway for mutant secretory proteins alphal-antitrypsin $\mathrm{Z}$ and $\mathrm{S}$ is distinct from that for an unassembled membrane protein. $J$ Biol Chem 1996;271(22):13215-13220.

11. Wu Y, Whitman I, Molmenti E, et al. A lags in intracellular degradation of mutant alpha 1-antitrypsin correlates with the liver disease phenotype in homozygous PiZZ alpha 1-antitrypsin deficiency. Proc Natl Acad Sci USA. 1994;91(19):9014-9018.

12. Le A, Graham KS, Sifers RN. Intracellular degradation of the transportimpaired human PiZ alpha 1-antitrypsin variant. Biochemical mapping of the degradative event among compartments of the secretory pathway. J Biol Chem. 1990;265(23):14001-14007.

13. Le A, Ferrell GA, Dishon DS, et al. Soluble aggregates of the human PiZ alpha 1-antitrypsin variant are degraded within the endoplasmic reticulum by a mechanism sensitive to inhibitors of protein synthesis. $J$ Biol Chem. 1992;267(2):1072-1080.

14. Hidvegi T, Ewing M, Hale P, et al. An autophagy-enhancing drug promotes degradation of mutant alphal-antitrypsin $\mathrm{Z}$ and reduces hepatic fibrosis. Science. 2010;329(5988):229-232.

15. Kamimoto T, Shoji S, Hidvegi T, et al. Intracellular inclusions containing mutant alpha1-antitrypsin $\mathrm{Z}$ are propagated in the absence of autophagic activity. J Biol Chem. 2006;281(7):4467-4476.

16. Kruse KB, Brodsky JL, McCracken AA. Characterization of an ERAD gene as VPS30/ATG6 reveals two alternative and functionally distinct protein quality control pathways: one for soluble $\mathrm{Z}$ variant of human alpha-1 proteinase inhibitor (A1PiZ) and another for aggregates of A1PiZ. Mol Biol Cell. 2006;17(1): 203-212.

17. Eggenschwiler R, Loya K, Sgodda M, et al. Hepatic differentiation of murine disease-specific induced pluripotent stem cells allows disease modelling in vitro. Stem Cells Int. 2011;2011: 924782.

18. Tafaleng EN, Chakraborty S, Han B, et al. Induced pluripotent stem cells model personalized variations in liver disease resulting from alpha1antitrypsin deficiency. Hepatology. 2015;62(1):147-157.

19. Wilson AA, Ying L, Liesa M, et al. Emergence of a stage-dependent human liver disease signature with directed differentiation of alpha-1 antitrypsin-deficient IPS cells. Stem Cell Reports. 2015;4(5):873-885.

20. Eggenschwiler R, Loya K, Wu G, et al. Sustained knockdown of a disease-causing gene in patient-specific induced pluripotent stem cells using lentiviral vector-based gene therapy. Stem Cells Transl Med. 2013;2(9):641-654.

21. Papp E, Száraz P, Korcsmáros T, et al. Changes of endoplasmic reticulum chaperone complexes, redox state, and impaired protein disulfide reductase activity in misfolding alpha1-antitrypsin transgenic mice. FASEB J. 2006;20(7):1018-1020.
22. Carlson JA, Rogers BB, Sifers RN, et al. Accumulation of PiZ alpha 1 -antitrypsin causes liver damage in transgenic mice. J Clin Invest. 1989;83(4):1183-1190.

23. Sifers RN, Finegold MJ, Woo SL. Alpha-1-antitrypsin deficiency: accumulation or degradation of mutant variants within the hepatic endoplasmic reticulum. Am J Respir Cell Mol Biol. 1989;1(5):341-345.

24. Hubner RH, Leopold PL, Kiuru M, et al. Dysfunctional glycogen storage in a mouse model of alpha1-antitrypsin deficiency. sJ Respir Cell Mol Biol. 2009;40(2):239-247.

25. Teckman JH,AnJK, Loethen S, et al. Fasting in alpha1-antitrypsin deficient liver: constitutive [correction of consultative] activation of autophagy. Am J Physiol Gastrointest Liver Physiol. 2002;283(5):1156-1165.

26. Marcus NY, Brunt EM, Blomenkamp K, et al. Characteristics of hepatocellular carcinoma in a murine model of alpha-1-antitrypsin deficiency. Hepatol Res. 2010;40(6):641-653.

27. Mela M, Davies S, Verma S, et al. P90 $\alpha-1$ antitrypsin (a1AT) polymers cause extreme hepatocyte ageing. Gut. 2011;60:A41-A42.

28. Marcus NY, Blomenkamp K, Ahmad M, et al. Oxidative stress contributes to liver damage in a murine model of alpha-1-antitrypsin deficiency. Exp Biol Med (Maywood). 2012;237(10):1163-1172.

29. Rudnick DA, Liao Y, An JK, et al. Analyses of hepatocellular proliferation in a mouse model of alpha-1-antitrypsin deficiency. Hepatology. 2004;39(4):1048--1055.

30. Chu AS, Chopra KB, Perlmutter DH. Is severe progressive liver disease caused by alpha-1-antitrypsin deficiency more common in children or adults? Liver Transpl. 2016;22(7):886-94.

31. Ding J, Yannam GR, Roy-Chowdhury N, et al. Spontaneous hepatic repopulation in transgenic mice expressing mutant human alpha1-antitrypsin by wild-type donor hepatocytes. J Clin Invest. 2011;121(5):1930-1934.

32. Lindblad, Blomenkamp K, Teckman J. Alpha-1-antitrypsin mutant Z protein content in individual hepatocytes correlates with cell death in a mouse model. Hepatology. 2007;46(4):1228-1235.

33. Rudnick DA, Shikapwashya O, Blomenkamp K, et al. Indomethacin increases liver damage in a murine model of liver injury from alpha-1antitrypsin deficiency. Hepatology. 2006;44(4):976-982.

34. Chu AS, Perlmutter DH, Wang Y. Capitalizing on the autophagic response for treatment of liver disease caused by alpha-1-antitrypsin deficiency and other genetic diseases. Biomed Res Int. 2014;2014:459823.

35. Teckman JH, Perlmutter DH. Retention of mutant alpha(1)-antitrypsin Z in endoplasmic reticulum is associated with an autophagic response. $\mathrm{Am}$ J Physiol Gastrointest Liver Physiol. 2000;279(5):961-974.

36. Settembre C, Zoncu R, Medina DL, et al. A lysosome-to-nucleus signalling mechanism senses and regulates the lysosome via mTOR and TFEB. EMBO J. 2012;31(5):1095-1108.

37. Pastore N, Ballabio A, Brunetti-Pierri N. Autophagy master regulator TFEB induces clearance of toxic SERPINA1/alpha-1-antitrypsin polymers. Autophagy. 2013;9(7):1094-1096.

38. Kaushal S, Annamali M, Blomenkamp K, et al. Rapamycin reduces intrahepatic alpha-1-antitrypsin mutant $\mathrm{Z}$ protein polymers and liver injury in a mouse model. Exp Biol Med (Maywood). 2010;235(6):700-709.

39. Li J, Pak SC, O’Reilly LP, et al. Fluphenazine reduces proteotoxicity in C. Elegans and mammalian models of alpha-1-antitrypsin deficiency. PLoS One. 2014;9(1):e87260.

40. Tang Y, Fickert P, Trauner M, et al. Autophagy Induced by Exogenous Bile Acids is Therapeutic in a Model of Alpha-1-AT Deficiency Liver Disease. Am J Physiol Gastrointest Liver Physiol. 2016;311(1):G156-65. 
41. Gosai SJ, Kwak JH, Luke CJ, et al. Automated high-content live animal drug screening using $\mathrm{C}$. elegans expressing the aggregation prone serpin alpha1-antitrypsin Z. PLoS One. 2010;5(11):e15460.

42. Long OS, Benson JA, Kwak JH, et al. A C. elegans model of human alpha1-antitrypsin deficiency links components of the RNAi pathway to misfolded protein turnover. Hum Mol Genet. 2014;23(19):5109-5122.

43. ClinicalTrials.gov. Alpha-1 Antitrypsin Deficiency Adult Liver Study, St. Louis University, UK; 2016.

44. Brantly ML, Spencer LT, Humphries M, et al. Phase I trial of intramuscular injection of a recombinant adeno-associated virus serotype 2 alphal-antitrypsin (AAT) vector in AAT-deficient adults. Hum Gene Ther. 2006;17(12):1177-1186.

45. Flotte TR, Brantly ML, Spencer LT, et al. Phase I trial of intramuscular injection of a recombinant adeno-associated virus alpha 1-antitrypsin (rAAV2-CB-hAAT) gene vector to AAT-deficient adults. Hum Gene Ther. 2004;15(1):93-128.

46. Lu Y, Choi YK, Campbell TM, et al. Therapeutic level of functional human alpha 1 antitrypsin (hAAT) secreted from murine muscle transduced by adeno-associated virus (rAAV1) vector. J Gene Med. 2006;8(6):730-735.

47. Song S, Morgan M, Ellis T, et al. Sustained secretion of human alpha-1antitrypsin from murine muscle transduced with adeno-associated virus vectors. Proc Natl Acad Sci. 1998;95(24):14384-14388.
48. Burrows JA, Willis LK, Perlmutter DH. Chemical chaperones mediate increased secretion of mutant alpha 1-antitrypsin (alpha 1-AT) Z A potential pharmacological strategy for prevention of liver injury and emphysema in alpha 1-AT deficiency. Proc Natl Acad Sci USA 2000;97(4):1796-1801

49. Teckman JH. Lack of effect of oral 4-phenylbutyrate on serum alpha-1antitrypsin in patients with alpha-1-antitrypsin deficiency: a preliminary study. J Pediatr Gastroenterol Nutr. 2004;39(1):34-37.

50. Guo S, Booten SL, Watt A, et al. Using antisense technology to develop a novel therapy for alpha-1 antitrypsin deficient (AATD) liver disease and to model AATD lung disease. Rare Dis. 2014;2:e28511.

51. Guo S, Booten SL, Aghajan M, et al. Antisense oligonucleotide treatment ameliorates alpha-1 antitrypsin-related liver disease in mice. $J$ Clin Invest. 2014;124(1):251-261.

52. Wooddell CI, Blomenkamp KS, Kanner S, et al. A hepatocyte-targeted RNAi-based treatment for liver disease associated with alpha-1antitrypsin deficiency [Abstract]. Hepatology. 2014;60:252.

53. Mueller C, Tang Q, Gruntman A, et al. Sustained miRNA-mediated knockdown of mutant AAT with simultaneous augmentation of wildtype AAT has minimal effect on global liver miRNA profiles. Mol Ther 2012;S20(3):590-600.

54. Wewers MD, Gadek JE, Keogh BA, et al. Evaluation of danazol therapy for patients with PiZ alpha-1-antitrypsin deficiency. Rev Respir Dis $1986 ; 134(3): 476-480$ 